\title{
THE CONVERGENCE OF SEQUENCES OF RATIONAL FUNCTIONS OF BEST APPROXIMATION. III $\left({ }^{1}\right)$
}

\author{
BY \\ J. L. WALSH
}

The present paper is a continuation of several papers [1]-[4] on the same general topic, and serves to give both details of some results previously mentioned without proof and further refinements of results already set forth. A function of type $(j, k)$ is defined as a rational function of the form

$$
\frac{a_{0} z^{j}+a_{1} z^{j-1}+\cdots+a_{j}}{b_{0} z^{k}+b_{1} z^{k-1}+\cdots+b_{k}}, \quad \sum\left|b_{i}\right| \neq 0
$$

and we study convergence as $n \rightarrow \infty$ of approximating functions of both types $(n, \nu)$ and $(\nu, n)$, where $\nu$ is constant. Our new results are concerned not primarily with Tchebycheff norms

$$
\|\phi(z)\|_{\infty}=[\max |\phi(z)|, \quad z \text { on } E]
$$

as in the former papers, but more especially with norms $(q>0)$ involving line integrals

$$
\|\phi(z)\|_{q}=\left[\int_{C}|\phi(z)|^{q}|d z|\right]^{1 / q}
$$

taken over the boundary $C$ (assumed rectifiable) of a given set $E$ and involving surface integrals

$$
\|\phi\|_{q}^{\prime}=\left[\iint_{E}|\phi(z)|^{q} d S\right]^{1 / q}
$$

taken over the closure $E$ of an open set. In $\S 1$ we study functions of type $(n, \nu)$ and in $\$ 2$ functions of type $(\nu, n)$, with reference to norms of both types (2) and (3), for approximation on $E$ to a function $f(z)$ analytic on $E$, meromorphic in a region containing $E$; we relate greatest geometric degree of convergence to regions in which $f(z)$ is meromorphic with a limited number of poles. In $\S \S 3$ and 4 we study the type functions as approximants, to given functions with more refined properties on specified sets containing $E$, namely functions of classes $H(k, \alpha, p)$; we relate degree of convergence more refined than geometric degree to boundary behavior of $f(z)$ in regions in which $f(z)$ is meromorphic.

Presented to the Society, November 26, 1965; received by the editors December 15, 1965.

(1) Research sponsored (in part) by the Air Force Office of Scientific Research. 
In the situations used below, for each norm and for each type there exists a rational function of best approximation to the given function on $E$. This extremal function need not be unique, but any extremal function suffices for each theorem.

1. Approximating functions of type $(n, v)$. Our fundamental theorem [1]-[4] is

THEOREM 1. In the z-plane let E be a closed bounded point set whose complement is connected, and regular in the sense that it possesses a Green's function $G(z)$ with pole at infinity. Let $\Gamma_{\sigma}$ denote generically the locus $G(z)=\log \sigma(>0)$, and $E_{\sigma}$ the (open) interior of $\Gamma_{\sigma}$. Let the function $f(z)$ be analytic on $E$, meromorphic with precisely $\nu$ poles (that is, poles of total order $v)$ in $E_{\rho}(1<\rho \leqq \infty)$. Let the rational functions $R_{n v}(z)$ of respective types $(n, \nu)$ satisfy for the norm on $E$

$$
\limsup _{n \rightarrow \infty}\left\|f(z)-R_{n v}(z)\right\|_{\infty}^{1 / n} \leqq 1 / \rho ;
$$

this condition is satisfied by the functions $R_{n v}(z)$ of best Tchebycheff approximation of type $(n, v)$ to $f(z)$ on $E$. Then for $n$ sufficiently large the function $R_{n v}(z)$ has precisely $\nu$ finite poles, which approach respectively the $\nu$ poles of $f(z)$ in $E_{\rho}$. If $D$ denotes the interior of $E_{\rho}$ with the $v$ poles of $f(z)$ deleted, the sequence $R_{n v}(z)$ converges to $f(z)$ throughout $D$, and for any closed set $S$ in $D$ and in the closed interior of $E_{\sigma}, 1<\sigma<\rho$, but not wholly in $E$, we have

$$
\limsup _{n \rightarrow \infty}\left[\max \left|f(z)-R_{n v}(z)\right|, \quad z \text { on } S\right]^{1 / n} \leqq \sigma / \rho .
$$

Thus far the $R_{n v}(z)$ need not be defined for every $n$, but below they shall be so defined. Whether the $R_{n v}(z)$ are extremal or not, let $\rho$ be the largest number such that $f(z)$ is meromorphic with precisely $v$ poles in $E_{\rho}$. If (4) holds, then (4) holds with the equality sign, as does (5) if $S$ is a continuum (not a single point) and $\sigma$ is chosen as small as possible.

Theorem 1 is to be regarded as relatively complete, in consideration of the weakness of the hypothesis on $f(z)$. It expresses (i) the asymptotic behavior of the finite poles of the $R_{n v}(z)$, (ii) the exact geometric degree of convergence on $E$, (iii) the region or regions of convergence of the sequence $R_{n v}(z)$ and the geometric degree of convergence on subsets of those regions. In connection with exact regions of convergence, we refer to [3, remarks after Corollary to Theorem 4].

The case $\nu=0$ of Theorem 1 refers to approximation to $f(z)$ on $E$ by polynomials, a subject on which the literature is extensive. Our conditions on the set $E$ are here the standard conditions for polynomial (Tchebycheff) approximation; conditions to be used below depend on the various norms: (1), (2), (3).

In the papers [2], [3] we chose to consider the norm (2) as fundamental, and from the corresponding results derived Theorem 1 (for the Tchebycheff norm); in the present paper, as in [4], the reverse procedure is more convenient. In the proof of Theorem 1 the primary tool is Lemma 1, now presented in a slightly more general form than that of [4], in that the finite poles of the $R_{n v}(z)$ are not required to be 
uniformly bounded; the more general form is proved [6] nevertheless by methods developed in [4].

LEMMA 1. With the notation and hypothesis on $E$ of Theorem 1, let rational functions $r_{n v}(z)$ of respective types $(n, \nu)$ satisfy the inequality

$$
\limsup _{n \rightarrow \infty}\left\|r_{n v}(z)\right\|_{\infty}^{1 / n} \leqq 1 / \rho_{1}, \quad 1<\rho_{1} \leqq \infty
$$

where $\nu$ is constant. Let $S$ be a closed set in the closed interior of $E_{\sigma}, 1<\sigma<\rho_{1}$, and containing no limit point of the poles of the $r_{n v}(z)$. Then the sequence $r_{n v}(z)$ converges uniformly to zero on $S$, and we have

$$
\limsup _{n \rightarrow \infty}\left[\max \left|r_{n v}(z)\right|, \quad z \text { on } S\right]^{1 / n} \leqq \sigma / \rho_{1} .
$$

We refer merely to [2]-[4] for details of the proof of the analogues of Theorem 1 ; compare [3, Theorem 8$]$ :

THEOREM 2. Let the point set E be closed, bounded, with rectifiable boundary, whose complement is connected, and consist of a finite number of components each satisfying those same conditions. Let $f(z)$ satisfy the conditions of Theorem 1, and also the $R_{n v}(z)$, except that (4) is replaced by

$$
\limsup _{n \rightarrow \infty}\left\|f(z)-R_{n v}(z)\right\|_{q}^{1 / n} \leqq 1 / \rho, \quad q>0 ;
$$

this condition is valid for the functions $R_{n v}(z)$ of least qth power approximation (2) on $E$. Then the conclusion of Theorem 1 holds.

THEOREM 3. Let the point set E consist of a finite number of components, each of which is a closed region whose complement is connected. Let $f(z)$ satisfy the conditions of Theorem 1, and also the $R_{n v}(z)$, except that (4) is replaced by

$$
\limsup _{n \rightarrow \infty}\left\|f(z)-R_{n v}(z)\right\|_{q}^{1 / n} \leqq 1 / \rho, \quad q>0 ;
$$

this condition is valid for the functions $R_{n v}(z)$ of least qth power approximation (3) on $E$. Then the conclusion of Theorem 1 holds.

2. Approximating functions of type $(\nu, n)$. Here the fundamental theorem [1], a consequence of Theorem 1 , is derived by setting $f(z) \equiv 1 / F(z)$.

THEOREM 4. Let $E$ be as in Theorem 1, and let the function $F(z)$ be analytic and different from zero on $E$, meromorphic with precisely $\nu$ zeros in $E_{\tau}, 1<\tau \leqq \infty$. Let the rational functions $R_{v n}(z)$ of respective types $(\nu, n)$ satisfy for the norm on $E$

$$
\limsup _{n \rightarrow \infty}\left\|F(z)-R_{v n}(z)\right\|_{\infty}^{1 / n} \leqq 1 / \tau ;
$$

this includes the functions $R_{v n}(z)$ of best Tchebycheff approximation on $E$. Then for $n$ sufficiently large the function $R_{v n}(z)$ has precisely $v$ finite zeros, which approach respectively the $\nu$ zeros of $F(z)$ in $E_{\tau}$. The poles of the $R_{v n}(z)$ interior to $E_{\tau}$ approach the 
respective poles of $F(z)$ interior to $E_{\imath}$, multiplicities included, and have no other limit point interior to $E_{\tau}$. If $D_{1}$ denotes the interior of $E_{\tau}$ with the poles of $F(z)$ deleted, the sequence $R_{v n}(z)$ converges to $F(z)$ throughout $D_{1}$, and for any closed set $S$ in $D_{1}$ and in the closed interior of $E_{\sigma}, 1<\sigma<\tau$, but not wholly in $E$, we have

$$
\limsup _{n \rightarrow \infty}\left[\max \left|f(z)-R_{v n}(z)\right|, \quad z \text { on } S\right]^{1 / n} \leqq \sigma / \tau .
$$

Thus far the $R_{v n}(z)$ need not be defined for every $n$, but below they shall be so defined.

Whether the $R_{v n}(z)$ are extremal or not, let $\tau$ be the largest number such that $F(z)$ is meromorphic with precisely $\nu$ zeros interior to $E_{\tau}(1<\tau \leqq \infty)$. If $(9)$ holds, then (9) holds with the equality sign, as does $(10)$ with $S=\Gamma_{\sigma}(1<\sigma<\tau)$, and provided no pole of $F(z)$ lies on $\Gamma_{\sigma}$.

Theorems 1 and 4 are the duals of each other, namely with the interchange of allowable zeros and poles, for both functions approximated and the approximating rational functions.

The remark that the poles of the $R_{v n}(z)$ can have no limit point in $E_{\tau}$ other than the poles of $F(z)$ follows because if a sequence of the $R_{v n}(z)$ has a corresponding sequence of poles approaching a point $z_{0}$ of analyticity of $F(z)$ in $E_{\rho}$, then in some annulus with $z_{0}$ as center the convergence of some subsequence is uniform. It follows (principle of argument) that $z_{0}$ is a limit also of zeros of the $R_{v n}(z)$, which is impossible. Likewise if a sequence of the $R_{v n}(z)$ has a corresponding sequence of sets of poles approaching a pole of $F(z)$ in $E_{\rho}$, the total order of the sets of poles must be the same (for $n$ sufficiently large) as the order of the pole of $F(z)$.

We shall establish the analogue of Theorem 4 for the norm (2), namely the dual of Theorem 2:

THEOREM 5. Let the point set $E$ consist of a finite number of mutually exterior closed Jordan regions $J_{1}, J_{2}, \ldots, J_{\mu}$, each with rectifiable boundary. Let $F(z)$ satisfy the conditions of Theorem 4, except that (9) is replaced by

$$
\limsup _{n \rightarrow \infty}\left\|F(z)-R_{v n}(z)\right\|_{q}^{1 / n} \leqq 1 / \tau, \quad q>0,
$$

where the $R_{v n}(z)$ have no limit point of poles on $E$. Then the conclusion of Theorem 4 holds.

Let the point set $E^{\prime}$ be an arbitrary closed set interior to $E$. By a convenient lemma $[5, \S 5.5$, extension of Lemma], [3, Lemma 2] we have from (11)

$$
\limsup _{n \rightarrow \infty}\left[\max \left|F(z)-R_{v n}(z)\right|, z \text { on } E^{\prime}\right]^{1 / n} \leqq 1 / \tau .
$$

Theorem 4 now applies, with $E$ replaced by $E^{\prime}$ where $E^{\prime}$ is suitably chosen (see below), and we conclude by (10)

$$
\limsup _{n \rightarrow \infty}\left[\max \left|F(z)-R_{v n}(z)\right|, z \text { on }\left(E^{\prime}\right)_{\sigma}\right]^{1 / n} \leqq \sigma / \tau .
$$


The level loci $\Gamma_{\sigma}$ of Green's function for the complement of $E$ with pole at infinity are monotonic with respect to the point set $E$, in the sense that if $E^{\prime}$ is a proper subset (whose complement is connected and regular) of $E$, then for $\sigma>1$ the closure of the point set $\left(E^{\prime}\right)_{\sigma}$ lies interior to $E_{\sigma}$. We choose $\sigma(>1)$ so small that the closure of $E_{\sigma}$ contains no zero or pole of $F(z)$. Then [5, $\$ 2.1$, Theorem 2] $J_{k}^{\prime}$ can be chosen as a closed Jordan region interior to $J_{k}$ so that $J_{k}$ lies interior to $\left(J_{k}^{\prime}\right)_{\sigma}$, and thus $E$ lies interior to $\sum\left[\left(J_{k}^{\prime}\right)_{\sigma}\right]$, which lies interior to $\left(E^{\prime}\right)_{\sigma}$, where $E^{\prime}=\sum J_{k}^{\prime}$; moreover the closure of $\left(E^{\prime}\right)_{\sigma}$ contains no zero or pole of $F(z)$. We choose $E^{\prime}$ so large also that $\left(E^{\prime}\right)_{\tau}=\left(E_{\sigma}^{\prime}\right)_{\tau / \sigma}$ contains the $\nu$ zeros of $F(z)$ in $E_{\tau}$, which justifies the derivation of (13) from (12). Consequently (13) implies the corresponding inequality for $z$ on $E$, and approach of $\sigma$ to unity yields (9). Now Theorem 4 applies without substitution, and Theorem 5 is established.

In Theorems 1 and 2 the approximating rational functions have each but a bounded number of finite poles, and those poles can be accounted for by the methods developed, whereas in Theorems 4 and 5 the approximating rational functions have each but a finite number of zeros, and those zeros can be accounted for; but in the latter case the behavior of the finite poles (perhaps unbounded in number) is not always easily determined.

In Theorem 5 we have deliberately excluded the case that $E$ is a Jordan arc, or that a component of $E$ is a Jordan arc. It is not known whether the theorem holds without that exclusion. It is quite conceivable that otherwise limit points of the poles of the given $R_{v n}(z)$ might be on the Jordan arc, indeed everywhere dense on the Jordan arc. Such poles cannot be treated by the methods just used. There are indications in the literature (Gončar [12]; Newman [13]) that for approximation on a Jordan $\operatorname{arc} E$, namely an interval of the axis of reals, degree of approximation by means of rational functions may be larger (approximation closer) when some or all of the poles approach $E$ than when no limit point of poles lies on $E$. This comment does not apply, as in Theorem 2, when poles of the approximating rational functions conceivably approaching $E$ are finite in number.

However, if we modify the hypothesis of Theorem 5 so that we suppose the poles of the given $R_{v n}(z)$ to have no limit point on $E$, then the conclusion remains valid when $E$ has a finite number of components each of which is a closed rectifiable Jordan arc or a closed Jordan region with rectifiable boundary. The proof of the modified Theorem 5 may be given by use of [3, Lemma 1].

Theorem 5 has an analogue where the norm (2) is replaced by (3):

THEOREM 6. Let the point set E consist of a finite number of components, each the closure of a simply connected region and the complement of an infinite region. Let $F(z)$ satisfy the conditions of Theorem 4, except that (9) is replaced by

$$
\limsup _{n \rightarrow \infty}\left\|F(z)-R_{v n}(z)\right\|_{q}^{1 / n} \leqq 1 / \tau, \quad q>0,
$$

where the $R_{v n}(z)$ have no poles on $E$. Then the conclusion of Theorem 4 holds. 
If $E^{\prime}$ is an arbitrary closed set in $E$, (14) implies (12) by virtue of [5, $§ 5.3$, Lemma II], and the remainder of the proof of Theorem 6 follows the remainder of the proof of Theorem 5 .

It is not without interest to consider a special case of approximation by functions of type $(\nu, n)$; we choose $F(z) \equiv z-c, c>1, E:|z| \leqq 1, \nu=0$. We obtain approximating functions (not necessarily extremal) with the proper degree of approximation by setting $R_{v n}(z) \equiv 1 / q_{n}(z)$,

$$
q_{n}(z) \equiv-\frac{1}{c}\left(1+\frac{z}{c}+\frac{z^{2}}{c^{2}}+\cdots+\frac{z^{n}}{c^{n}}\right) \equiv \frac{1-z^{n+1} / c^{n+1}}{z-c}
$$

The formula for suitably chosen $z$ on $|z|=1$ :

$$
\left|F(z)-R_{v n}(z)\right| \equiv\left|(z-c)-\frac{z-c}{1-z^{n+1} / c^{n+1}}\right| \equiv\left|\frac{-z^{n+1} / c^{n+1}}{q_{n}(z)}\right|
$$

gives the supremum of the first member on $E$. We notice namely that $q_{n}(z)$ has $n$ zeros on the circle $|z|=c$, so the norm of the deviation on $|z|=c$ is infinite for all values of $n(>0)$. For the Taylor development, Jentzsch's theorem asserts that each point of the circle of convergence is a limit point of zeros of the partial sums; the reciprocals $1 / q_{n}(z)$ of the partial sums (in the present example) have poles on that circle, of which every point of the circle of convergence is a limit point.

3. Approximating functions of type $(n, v)$, refined degree of convergence. In Theorems 1-5 we have considered geometric degree of convergence, which is closely related to regions of meromorphicity of $f(z)$, and now proceed to study more refined measures of convergence, related to boundary behavior of functions in such a region of meromorphicity.

We shall deal on $\Gamma_{\rho}$ with classes of functions $H(k, \alpha, p)$ for $0<\alpha<1$ and $Z(k, p)$ where we set $\alpha=1$, which for $k \geqq 0$ are classes of functions with bounded norms on level loci, having on $\Gamma_{o}$ boundary values whose $k$ th derivatives satisfy a $p$ th power integrated Lipschitz condition of order $\alpha$ for $0<\alpha<1$ and an integrated Zygmund condition for $\alpha=1$; further details are found in [9].

There has been established $[4, \S 7]$ a first result here, namely Theorem 7 for the case $p=\infty$ :

THEOREM 7. Let the point set $E$ consist of a finite number of mutually exterior closed regions, each bounded by an analytic Jordan curve, and let $f(z)$ be analytic on $E$, meromorphic with precisely $\nu$ poles on $E_{\rho}$, and with $1 \leqq p \leqq \infty$ of class $H(k, \alpha, p)$ for $0<\alpha<1$ or of class $Z(k, p)$ for $\alpha=1$ on $\Gamma_{\rho}$. If $\Gamma_{\rho}$ has no multiple points, then there exist rational functions $R_{n v}(z)$ of respective types $(n, v)$ such that we have

$$
\left|f(z)-R_{n v}(z)\right| \leqq A / n^{k+\alpha} \rho^{n}, \quad z \text { on } E ;
$$

here and below the number $A$ is constant (independent of $n$ and $z$ ) but may vary from 
one inequality to another. Moreover we have for an arbitrary sequence $R_{n v}(z)$ satisfying (15) also

$$
\left|f(z)-R_{n v}(z)\right| \leqq A \sigma^{n} / n^{k+\alpha} \rho^{n}, \quad z \text { on } S,
$$

where $S$ is an arbitrary closed set in the closed interior of $\Gamma_{\sigma}, 1<\sigma<\rho$, containing no pole of $f(z)$; we have in addition

$$
\left|f(z)-R_{n v}(z)\right| \leqq A / n^{k+\alpha-1}, \quad z \text { on } \Gamma_{\rho},
$$

provided $k \geqq 1$, so if $\Gamma_{\rho}$ has no multiple points $f(z)$ is of class $H(k-1, \alpha, \infty)$ on $\Gamma_{\rho}$ if $0<\alpha<1$ and of class $Z(k-1, \infty)$ if $\alpha=1$.

It is sufficient here [7] if the Jordan curves bounding $E$ are not analytic, but have a parametric representation $x=x(s), y=y(s)$ with respect to arc length $s$ where the functions have continuous second derivatives satisfying a Lipschitz condition of some positive order. The primary question in this connection is the uniform boundedness on $E$ of the polynomials $\omega_{n}(z) \equiv z^{n+1}+\cdots$; the zeros of $\omega_{n}(z)$ are (if $k \geqq 0$ ) the $n+1$ points of interpolation on $E$ of the polynomial $p_{n}(z)$ to the function $\pi(z) f(z)$ minus aǹ auxiliary polynomial in (18); compare also Lemma 4 below. We do not restrict $k$ to positive or nonnegative values.

Let $\pi(z)$ denote the polynomial $z^{\nu}+a_{1} z^{\nu-1}+\cdots$, whose zeros are the $\nu$ poles of $f(z)$ interior to $\Gamma_{\rho}$. Since $\pi(z)$ is analytic on $\Gamma_{\rho}$, the function $\pi(z) f(z)$ is [9] of class $H(k, \alpha, p)$ or $Z(k, p)$ on $\Gamma_{\rho}$, analytic in $E_{\rho}$, and there exist [9] polynomials $p_{n}(z)$ of respective degrees $n$ such that we have

$$
\left|\pi(z) f(z)-p_{n}(z)\right| \leqq A / n^{k+\alpha} \rho^{n}, \quad z \text { on } E .
$$

Consequently we may write

$$
\left|f(z)-p_{n}(z) / \pi(z)\right| \leqq A / n^{k+\alpha} \rho^{n}, \quad z \text { on } E,
$$

and the second function between bars is a rational function $R_{n v}(z)$ of type $(n, v)$ :

$$
\left\|f(z)-R_{n v}(z)\right\| \leqq A / n^{k+\alpha} \rho^{n}, \quad z \text { on } E .
$$

A fortiori the rational functions of type $(n, v)$ of best (Tchebycheff) approximation to $f(z)$ on $E$ satisfy this same inequality.

By the well-known properties $[5, \S 4.6]$ of the polynomials $p_{n}(z)$ in $(18)$ we have $(1<\sigma<\rho)$

$$
\begin{aligned}
\left|\pi(z) f(z)-p_{n}(z)\right| & \leqq A \sigma^{n} / n^{k+\alpha} \rho^{n}, \quad z \text { on } E_{\sigma}+\Gamma_{\sigma}, \\
\left|f(z)-R_{n v}(z)\right| & \leqq A \sigma^{n} / n^{k+\alpha} \rho^{n}, \quad z \text { on } S .
\end{aligned}
$$

If the $r_{n v}(z)$ are arbitrary functions of type $(n, v)$ satisfying (19), we have by (19)

$$
\left|r_{n v}(z)-R_{n v}(z)\right| \leqq A / n^{k+\alpha} \rho^{n}, \quad z \text { on } E,
$$

and by the method of proof of Lemma 1

$$
\left|r_{n v}(z)-R_{n v}(z)\right| \leqq A \sigma^{n} / n^{k+\alpha} \rho^{n}, \quad z \text { on } S .
$$


Inequality (20) now yields by (21)

$$
\left|f(z)-r_{n v}(z)\right| \leqq A \sigma^{n} / n^{k+\alpha} \rho^{n}, \quad z \text { on } S,
$$

which is (16). Inequality (17) follows merely from (15) if we write

$$
\left|R_{n+1, v}(z)-R_{n v}(z)\right| \leqq A / n^{k+\alpha} \rho^{n}, \quad z \text { on } E,
$$

and deduce (again by the method of proof of Lemma 1)

$$
\left|R_{n+1, v}(z)-R_{n v}(z)\right| \leqq A / n^{k+\alpha}, \quad z \text { on } E_{\rho}\left({ }^{2}\right) .
$$

The functions $R_{n v}(z)$ have no poles in some neighborhood of $\Gamma_{\rho}$, and by the method of proof of Lemma 1 satisfy an inequality of form $\left|R_{n v}(z)\right| \leqq A \rho_{2}^{n}, \rho_{2}>1$, in such a neighborhood. The remaining part of Theorem 7 now follows from (17) and [9, Theorem 8].

Theorem 7 has immediate application to the study of least $q$ th power approximation to $f(z)$ on $E$; inequality (22) follows from (15):

THEOREM 8. Under the conditions of Theorem 7 , if $\Gamma_{\rho}$ has no multiple points, we have for the least qth power functions $R_{n v}(z)$ to $f(z)$ on $E$

$$
\left\|f(z)-R_{n v}(z)\right\|_{q} \leqq A / n^{k+\alpha} \rho^{n}, \quad q>0,
$$

where the norm involves a line integral over the boundary of E. For these extremal functions we also have

$$
\begin{aligned}
& \left|f(z)-R_{n v}(z)\right| \leqq A / n^{k+\alpha-1 / q} \rho^{n}, \quad z \text { on } E, \\
& \left|f(z)-R_{n v}(z)\right| \leqq A \sigma^{n} / n^{k+\alpha} \rho^{n}, \quad z \text { on } S,
\end{aligned}
$$

and if $k \geqq 1$

$$
\left|f(z)-R_{n v}(z)\right| \leqq A / n^{k+\alpha-1}, \quad z \text { on } \Gamma_{\rho} .
$$

Lemma 1 has an analogue [3, Lemma 1 , slightly extended] whose hypothesis is more precise than (6) for the $q$ th power norm on $E$ and whose conclusion is correspondingly more precise than that of Lemma 1 on $S$. The method of proof of that analogue, applied to the inequality

$$
\left\|R_{n+1, v}(z)-R_{n v}(z)\right\|_{q} \leqq A / n^{k+\alpha} \rho^{n},
$$

which is a consequence of (22), gives at once both (24) and (25). Indeed, (24) and (25) follow from (22) whether the $R_{n v}(z)$ are extremal or not.

It remains to prove (23), which is done by applying results due to D. Jackson [10]. Jackson studies a sequence of polynomials $p_{n}(z)$ of respective degrees $n$ with the notation

$$
\left[\max \left|f(z)-p_{n}(z)\right|, \quad z \text { on } C\right]=\varepsilon_{n}, \quad \varepsilon_{n} \rightarrow 0,
$$

$\left({ }^{2}\right)$ The writer withdraws the statement $[4, \S 7,(38)]$. With the notation of [4], there exist polynomials $p_{n}(z)$ satisfying (32), and there exist polynomials $p_{n}(z)$ satisfying (36) with $\sigma=\rho$; it is not known whether there exist polynomials $p_{n}(z)$ satisfying both inequalities simultaneously. 
where $C$ is a Jordan curve $x=x(s), y=y(s)$ referred to arc length as parameter, and where $x^{\prime \prime}(s)$ and $y^{\prime \prime}(s)$ exist everywhere on $C$ and are bounded. He then shows that for the corresponding polynomials $P_{n}(z)$ of respective degrees $n$ of least $q$ th power approximation to $f(z)$ on $C$ we have $(q>0)$

$$
\left|f(z)-P_{n}(z)\right| \leqq A n^{1 / q} \varepsilon_{n}, \quad z \text { on } C .
$$

The proof of (27) involves algebraic inequalities standard in the theory of approximation by real functions plus the lemma (Bernstein) that if a polynomial $P(z)$ of degree $n$ satisfies the inequality $|P(z)| \leqq L$ in $|z| \leqq 1$, then $\left|P^{\prime}(z)\right| \leqq n L$ in $|z| \leqq 1$.

In Theorem 8 the role of Jackson's $p_{n}(z)$ is taken by the $R_{n v}(z)$ of Theorem 7 , with (15) and (22); the role of Jackson's $P_{n}(z)$ is taken by the extremal $R_{n v}(z)$; the role of the lemma just mentioned is taken by

LEMMA 2. Let the function $R_{n+v, 2 v}(z) \equiv P_{n+v}(z) / Q_{2 v}(z)$ be of modulus not greater than $L$ in $|z| \leqq 1$, where the zeros of $Q_{2 v}(z)$ are bounded in modulus and bounded below in modulus from unity. Then in $|z| \leqq 1$ we also have

$$
\left|R_{n+v, 2 v}^{\prime}(z)\right| \leqq A L(n+v),
$$

where $A$ is a constant independent of $n$ and $z$.

We are dealing in Theorem 8 with functions $R_{n+v, 2 v}(z)$ equal to the extremal $R_{n v}(z)$ minus the $R_{n v}(z)$ of Theorem 7, for each of which latter functions the finite poles are known (Theorem 2) to approach the $\nu$ given poles of $f(z)$. Thus we have for $n$ sufficiently large

$$
0<M_{0} \leqq\left|Q_{2 v}(z)\right| \leqq M_{1}, \quad|z| \leqq 1, \quad Q_{2 v}(z) \equiv z^{2 v}+\cdots,
$$

where $M_{0}$ and $M_{1}$ are independent of $n$ and of $z$,

$$
\frac{\left|P_{n+v}(z)\right|}{M_{1}} \leqq\left|\frac{P_{n+v}(z)}{Q_{2 v}(z)}\right| \leqq L, \quad\left|P_{n+v}(z)\right| \leqq L M_{1}, \quad|z| \leqq 1
$$

We can now write

$$
\begin{aligned}
\left|R_{n+v, 2 v}^{\prime}(z)\right| & =\left|\frac{Q_{2 v}(z) P_{n+v}^{\prime}(z)-P_{n+v}(z) Q_{2 v}^{\prime}(z)}{Q_{2 v}^{2}(z)}\right| \\
& \leqq \frac{M_{1} \cdot L M_{1}(n+\nu)+2 L M_{1}^{2} \cdot \nu}{M_{0}^{2}} \leqq A L(n+v) .
\end{aligned}
$$

In the actual application of Lemma 2, we use inequality (28) not necessarily in the unit disk, but in a variable disk of constant radius which lies in $E$. In $E$ and in all such disks the $R_{n+v, 2 v}(z)$ are uniformly bounded, so (28) holds; the disks can be chosen to cover $E$.

The reasoning used by Jackson is valid in the present case, with $\varepsilon_{n}$ equal to the second member of (22), and yields (23).

Jackson points out that his methods yield also a result concerning approximation as measured by surface integrals, commencing with (26) but where in defining 
$P_{n}(z)$ the line integral is replaced by a surface integral; the conclusion (27) is valid with the exponent $1 / q$ replaced by $2 / q$. We still use Lemma 2 , and shall prove

THEOREM 9. Under the conditions of Theorem 7, if $\Gamma_{\rho}$ has no multiple points, we have for the least qth power functions $R_{n v}(z)$ to $f(z)$ on $E$

$$
\left\|f(z)-R_{n v}(z)\right\|_{a}^{\prime} \leqq A / n^{k+\alpha} \rho^{n}, \quad q>0,
$$

where the norm involves a surface integral over E. For these extremal functions we have also

$$
\begin{aligned}
& \left|f(z)-R_{n v}(z)\right| \leqq A / n^{k+\alpha-2 / q} \rho^{n}, \quad z \text { on } E, \\
& \left|f(z)-R_{n v}(z)\right| \leqq A \sigma^{n} / n^{k+\alpha-2 / a} \rho^{n}, \quad z \text { on } S,
\end{aligned}
$$

and if $k+\alpha-1-2 / q>0$,

$$
\left|f(z)-R_{n v}(z)\right| \leqq A / n^{k+\alpha-1-2 / q}, \quad z \text { on } \Gamma_{\rho} .
$$

If $q \geqq 1$, for the norm (2) on $E$ we have for those functions

$$
\begin{aligned}
\left\|f(z)-R_{n v}(z)\right\|_{q} & \leqq A / n^{k+\alpha-1 / q} \rho^{n}, \\
\left|f(z)-R_{n v}(z)\right| & \leqq A \sigma^{n} / n^{k+\alpha-1 / q} \rho^{n}, \quad z \text { on } S,
\end{aligned}
$$

and if $k+\alpha-1-1 / q>0$

$$
\left|f(z)-R_{n v}(z)\right| \leqq A / n^{k+\alpha-1-1 / q}, \quad z \text { on } \Gamma_{\rho} .
$$

Inequality (29) follows from (15), and (30) follows from (29) by the method of Jackson for surface integrals already mentioned; then (31) and (32) follow from (30) by the method of Lemma 1.

The proof of (33) follows a different order of ideas, and results namely from Lemma 3 below. By the methods of proof of Theorem 8, inequality (33) implies (34) and (35). It remains to establish (33), which we do by the methods of [11] and [8]. From [11, Theorem 9.5] we have

LEMMA 3. Let $C$ be an analytic Jordan curve of the z-plane whose interior is denoted by $D$, and let $P(z)$ be a polynomial in $z$ of degree $n$. Then we have for the norms on $C$ and $D$

$$
\|P(z)\|_{q} \leqq A_{0} n^{1 / q}\|P(z)\|_{q}^{\prime}, \quad q \geqq 1, n \geqq 1,
$$

where the constant $A_{0}$ is independent of $n$.

Under the conditions of Theorem 9, the finite poles of the $R_{n v}(z)$ approach the $\nu$ poles of $f(z)$ in $E_{\rho}$, by Theorem 3 , so for $n$ sufficiently large the polynomials $z^{v}+\cdots$ whose zeros are those poles are bounded and bounded from zero on $E$. Thus (36) with $A_{0}$ suitably adjusted is valid for those rational functions $R_{n v}(z)$ of type $(n, v)$, and for the difference of two such functions. For simplicity we denote $R_{n v}(z)$ by $p_{n}(z)$. 
Following a method due to de la Vallée Poussin used in [11] and [8], we write the series (convergent by Theorem 3 ) for $z$ on $E$

$$
f(z)-p_{n}(z) \equiv\left[p_{2^{m}}(z)-p_{n}(z)\right]+\left[p_{2^{m+1}}(z)-p_{2^{m}}(z)\right]+\left[p_{2^{m+2}}(z)-p_{2^{m+1}}(z)\right]+\cdots ;
$$

for given $n$ we choose $m$ so that $2^{m-1} \leqq n<2^{m}$. By (29), we have for the $q$ th power norms

$$
\begin{aligned}
\left\|f(z)-p_{2^{m}}(z)\right\|^{\prime} & \leqq A / 2^{m(k+\alpha)} \rho^{2^{m}}, \quad\left\|f(z)-p_{2^{m+1}}(z)\right\|^{\prime} \leqq A / 2^{(m+1)(k+\alpha)} \rho^{2^{m+1}}, \ldots ; \\
\left\|p_{2^{m}}(z)-p_{n}(z)\right\|^{\prime} & \leqq A_{1} / n^{k+\alpha} \rho^{n}, \quad\left\|p_{2^{m+1}}(z)-p_{2^{m}}(z)\right\|^{\prime} \leqq A_{1} / 2^{m(k+\alpha)} \rho^{2^{m}}, \ldots
\end{aligned}
$$

From Lemma 3 we have, for $q$ th power norms now over $C$,

$$
\begin{aligned}
\left\|p_{2^{m}}(z)-p_{n}(z)\right\| & \leqq A_{2} / n^{k+\alpha-1 / q} \rho^{n} \\
\left\|p_{2^{m+1}}(z)-p_{2^{m}}\right\| & \leqq A_{2} / 2^{m(k+\alpha)-1 / q} \rho^{2^{m}}, \ldots
\end{aligned}
$$

The sequence $p_{n}(z)$ converges in the $q$ th power mean on $C$ and also uniformly on the set $C+D$, by Theorem 3 , to a function analytic on and within $C$, a function necessarily by (29) and Theorem 3 identical to $f(z)$ there. Inequality (33) for every $n$ follows from inequalities (37), by the triangle inequality $(q \geqq 1)$, and the proof of Theorem 9 is complete.

It seems to the writer quite conceivable that the latter part of Theorem 9 is valid also if $q<1$, for Lemma 3 is valid [8] if $q<1$ and $C$ is a circular disk. If so, that result seems to lie considerably deeper than Theorem 9.

The theorems of $\S 3$ already established refer to $E$ as a relatively well-behaved point set, but partial extensions exist, as we proceed to indicate. If the point set $E$ is the line segment $(-1,+1)$, the locus $\Gamma_{R}$ is the ellipse whose foci are the ends of the segment and whose axes are $R+1 / R$ and $R-1 / R$. The shortest distance from a point of $E$ to a point of $E_{R}$ is the distance from the focus $z=1$ to the nearer vertex $z=(R+1 / R) / 2$ of the ellipse, namely $(R-1)^{2} / 2 R$. We say that a point set $E$ (closed, bounded) is of class $A$ provided $\delta(>0)$ exists such that each point $z_{0}$ of $E$ is contained in some line segment of length $\delta$ belonging wholly to $E$. In this case it follows from the monotonic character of the level locus $E_{R}$ as a function of (variable) $E$ that for a set of class $A$ the shortest distance from a point of $E$ to $E_{R}$ is not less than $(R-1)^{2} \delta / 2 R$.

Likewise if $E$ is the circular disk $|z|=\delta(>0)$, the locus $E_{R}$ is $|z|=\delta R$, and the shortest distance from a point of $E$ to $E_{R}$ is $\delta(R-1)$. We say that a closed bounded point set $E$ is of class $B$ provided $\delta(>0)$ exists so that each point of $E$ is contained in some closed circular disk of radius $\delta$ belonging wholly to $E$. Again it follows from the monotonic behavior of level loci that for a set $E$ of class $B$ the shortest distance from a point of $E$ to $E_{R}$ is not less than $(R-1) \delta$.

In the following lemma the Fekete points $[5, \S 7.7]$ are used, introduced by him in connection with the transfinite diameter of a given point set and later as points of interpolation; we have also borrowed some ideas from Shen $[5, \S 7.8]$. 
LEMMA 4. Let E be a closed bounded point set, whose complement is connected and regular, and of class $A$ or $B$ respectively. Let $\omega_{n}(z)$ be the polynomial $z^{n+1}+\cdots$ whose $n+1$ zeros are the $n+1$ Fekete points of order $n$ associated with $E$. Then for $z$ on $E$ and $t$ on $E_{R}(R>1)$ we have respectively

$$
\left|\omega_{n}(z) / \omega_{n}(t)\right| \leqq A n^{\lambda} / R^{n}, \quad \lambda=3 \text { or } 2,
$$

according as $E$ is of class $A$ or $B$.

If $p_{n}(z, t)$ denotes the polynomial of degree $n$ coinciding with the function $1 /(t-z)$ in the Fekete points of order $n$, where $t$ is not on $E$, we have $[5, \S 7.8]$ for $z$ on $E$ and for $t$ on $E_{1+1 / n}, n \geqq 1$,

$$
\left|p_{n}(z, t)\right| \leqq(n+1) / d_{1} \leqq 4 n^{2}(n+1) / \delta, \quad d_{1}=\operatorname{dist}\left[E, E_{1+1 / n}\right] \geqq \delta / 4 n^{2},
$$

where $E$ is of class $A$. From the identity

$$
1 /(t-z)-p_{n}(z, t) \equiv \omega_{n}(z) / \omega_{n}(t)(t-z)
$$

we deduce for $z$ and $t$ on these same sets

$$
\left|\omega_{n}(z) / \omega_{n}(t)(t-z)\right| \leqq 4 n^{2}(n+2) / \delta
$$

and if $d_{2}$ denotes the greatest distance from a point of $E$ to a point of $E_{R_{1}}$ with $R_{1}=2$,

$$
\left|\omega_{n}(z) / \omega_{n}(t)\right| \leqq 4 n^{2}(n+2) d_{2} / \delta, \quad n \geqq 1 .
$$

Let $w=\phi(z)$ denote the function analytic except perhaps for branch points which maps the complement of $E$ onto $|w|>1$ with $\phi(\infty)=\infty$; then the following inequality holds for $z$ fixed on $E$ and for all $t$ on $E_{1+1 / n}$, hence holds for $z$ fixed on $E$ and for all $t$ on or exterior to $E_{1+1 / n}$

$$
\begin{aligned}
\left|\frac{\omega_{n}(z)[\phi(t)]^{n+1}}{\omega_{n}(t)}\right| & \leqq 4 n^{2}(n+2) \frac{d_{2}}{\delta}\left(1+\frac{1}{n}\right)^{n+1}, \\
& \leqq 8 n^{2}(n+2) d_{2} e / \delta .
\end{aligned}
$$

In particular for $t$ on $E_{R}$ there follows $(R>1+1 / n)$

$$
\left|\omega_{n}(z) / \omega_{n}(t)\right| \leqq 8 n^{2}(n+2) d_{2} e / \delta R^{n+1},
$$

which implies (38) with $\lambda=3$. Point sets $E$ of class $B$ may be treated similarly.

THEOREM 10. Let $E$ be a point set of class $A$, and let $f(z)$ be analytic on $E$, meromorphic with precisely $\nu$ poles on $E_{\rho}$, and with $1 \leqq p \leqq \infty$, of class $H(k, \alpha, p)$ with $0<\alpha<1$ or of class $Z(k, p)$ with $\alpha=1$ on $\Gamma_{\rho}$. If $\Gamma_{\rho}$ has no multiple points, there exist rational functions $R_{n v}(z)$ of respective types $(n, v)$ such that we have for the norm on $E$

$$
\left\|f(z)-R_{n v}(z)\right\|_{\infty} \leqq A / n^{k+\alpha-3} \rho^{n} .
$$


Moreover for an arbitrary sequence $R_{n v}(z)$ satisfying (39) we have

$$
\left|f(z)-R_{n v}(z)\right| \leqq A \sigma^{n} / n^{k+\alpha-3} \rho^{n}, \quad z \text { on } S,
$$

where $S$ is an arbitrary closed set in the closed interior of $\Gamma_{\sigma}, 1<\sigma<\rho$, containing no pole of $f(z)$,

$$
\left|f(z)-R_{n v}(z)\right| \leqq A / n^{k+\alpha-4}, \quad z \text { on } \Gamma_{\rho},
$$

provided $k>3$.

If $E$ (of class A) consists of a finite number of mutually disjoint closed Jordan arcs, or Jordan regions, or both, with rectifiable boundary $C$, it follows from (39) that for the extremal functions $R_{n v}(z)$ of type $(n, v)$ with qth power norm (2) on $E$ we have $(q>0)$

$$
\left\|f(z)-R_{n v}(z)\right\|_{q} \leqq A / n^{k+\alpha-3} \rho^{n} ;
$$

for these same functions (40) and (41) hold. For arbitrary functions $R_{n v}(z)$ of types $(n, \nu)$ satisfying (42), inequalities (40) and (41) are valid, and if $k>3$ and $\Gamma_{\rho}$ has no multiple points, (41) implies that $f(z)$ is of class $H(k-4, \alpha, \infty)$ on $\Gamma_{\rho}$ if $0<\alpha<1$ and of class $Z(k-4, \infty)$ if $\grave{\alpha}=1$.

If $E$ consists of a finite number of mutually exterior closed Jordan regions, it follows from (39) that for the functions $R_{n v}(z)$ of best approximation to $f(z)$ on $E$ with qth power norm (3) we have

$$
\left\|f(z)-R_{n v}(z)\right\|_{q}^{\prime} \leqq A / n^{k+\alpha-3} \rho^{n} .
$$

It is clear that if class $A$ is replaced by class $B$ in Theorem 10, the powers of $n$ are increased by unity in (39), (40), (42), (43), and if $k>2$ also (41).

Inequality (39) follows from (38) by the usual methods [9, Problem $\beta$ ] applied to the function $\pi(z) f(z)$ used in the proof of Theorem 7, together with Lemma 4; the inequalities (40) and (41) follow from (42) as do (24) and (25) from (22) in the proof of Theorem 8 .

Theorem 10 seems to be new even in the case $\nu=0$, of approximation by polynomials.

4. Approximating functions of type $(\nu, n)$, refined degree of convergence. We return now to the topic considered in $\$ 2$, in the light of refinements of the sort developed in $\S 3$.

THEOREM 11. Let $E$ be as in Theorem 7, let the function $F(z)$ be analytic and different from zero on $E$, meromorphic with precisely $\nu$ zeros in $E_{\tau}, 1<\tau \leqq \infty$, and on $\Gamma_{\tau}$ let $f(z) \equiv 1 / F(z)$ be of class $H(k, \alpha, p),(1 \leqq p \leqq \infty)$, with $0<\alpha<1$ or of class $Z(k, p)$ with $\alpha=1$, where $\Gamma_{\tau}$ has no multiple points. Let the rational functions $R_{v n}(z)$ of respective types $(\nu, n)$ satisfy for the norm on $E$

$$
\left\|F(z)-R_{v n}(z)\right\|_{\infty} \leqq A / n^{k+\alpha} \tau^{n} ;
$$

this inequality is satisfied by the functions $R_{v n}(z)$ of best Tchebycheff approximation 
on $E$. If $D_{1}$ denotes the interior of $E_{\tau}$ with the poles of $F(z)$ deleted, on an arbitrary closed subset $S$ of $D_{1}$ contained in $E_{\sigma}, 1<\sigma<\tau$, we have

$$
\left|F(z)-R_{v n}(z)\right| \leqq A \sigma^{n} / n^{k+\alpha} \tau^{n}, \quad z \text { on } S .
$$

We have also

$$
\left|f(z)-1 / R_{v n}(z)\right| \leqq A / n^{k+\alpha-1}, \quad z \text { on } \Gamma_{\tau},
$$

provided $k \geqq 1$, so if $\Gamma_{\imath}$ has no multiple points, $f(z)$ is of class $H(k-1, \alpha, \infty)$ on $\Gamma_{\tau}$ if $0<\alpha<1$ and of class $Z(k-1, \infty)$ if $\alpha=1$.

By Theorem 7 for those functions $R_{n v}(z)$ of Theorem 7 and $n$ sufficiently large there follows $R_{n v}(z) \neq 0$ on $E$, and by (15)

$$
\left|1 / f(z)-1 / R_{n v}(z)\right| \leqq A / n^{k+\alpha} \tau^{n}, \quad z \text { on } E .
$$

This shows the existence of functions $R_{v n}(z) \equiv 1 / R_{n v}(z)$ of class $(\nu, n)$ which satisfy (44). Thus (44) is satisfied a fortiori by the functions of class $(\nu, n)$ of best Tchebycheff approximation.

If (44) is given, we may write conversely (15), where $R_{n v}(z) \equiv 1 / R_{v n}(z)$; inequality (16) follows for $z$ on the set $S_{1}$ found by deleting an open neighborhood of each zero of $F(z)$ on $S$, which implies (45) for $z$ on $S_{1}$, hence (45) follows for $z$ on $S$.

The remaining part of Theorem 11 follows from Theorem 7.

We proceed to consider norms (2) and (3) on $E$, in order.

THEOREM 12. Let E satisfy the conditions of Theorem 7. Let $f(z)$ and $F(z)$ be as in Theorem 11. Then there exist rational functions $R_{v n}(z)$ of respective types $(v, n)$ and having no poles on $E$, such that we have

$$
\left\|F(z)-R_{v n}(z)\right\|_{q} \leqq A / n^{k+\alpha} \tau^{n}, \quad q>0,
$$

namely the functions $R_{v n}(z)$ of least qth power approximation on $E$. For the Tchebycheff norm on $E$ and the extremal functions we have

$$
\left\|F(z)-R_{v n}(z)\right\|_{\infty} \leqq A / n^{k+\alpha-1 / q} \tau^{n} .
$$

If $D_{1}$ denotes the interior of $E_{\tau}$ with the poles of $F(z)$ deleted, on an arbitrary closed subset $S$ of $D_{1}$ contained in $E_{\sigma}, 1<\sigma<\tau$, we have for the extremal functions

$$
\left|F(z)-R_{v n}(z)\right| \leqq A \sigma^{n} / n^{k+\alpha-1 / q} \tau^{n}, \quad z \text { on } S .
$$

Likewise there follows if $k+\alpha-1-1 / q>0$ from (48) for an arbitrary sequence $R_{v n}(z)$

$$
\left|f(z)-1 / R_{v n}(z)\right| \leqq A / n^{k+\alpha-1-1 / q}, \quad z \text { on } \Gamma_{\tau},
$$

so if $\Gamma_{\imath}$ has no multiple points, then $f(z)$ is of class

$$
H([k+\alpha-1-1 / q], k+\alpha-1-1 / q-[k+\alpha-1-1 / q], \infty)
$$

or class

$$
Z([k+\alpha-1-1 / q], \infty)
$$


on $\Gamma_{\tau}$ according as $k+\alpha-1-1 / q-[k+\alpha-1-1 / q]$ is less than unity or equal to unity. Here $[Q]$ indicates the largest integer less than $Q$.

Inequality (47) follows from (44), (48) from (47) by the method of Jackson, and the remainder of the theorem follows from (48). It is the method of Jackson that is used here rather than his specific result (for approximation by polynomials). The important tool that we need is akin to Lemma 2, but refers to a function

$$
\Phi(z) \equiv P_{1}(z) / Q_{1}(z)-P_{2}(z) / Q_{2}(z),
$$

where the first quotient is the function $R_{v n}(z)$ of (44) and the second quotient is the function $R_{v n}(z)$ of (47). Each of these functions approaches (Theorem 5) $F(z)$ uniformly on $E$, the zeros of the functions approach those of $F(z)$ in $E_{\imath}$, and their poles in $E_{\tau}$ approach those of $F(z)$ in $E_{\tau}$. If we write $P_{1}(z) \equiv z^{v}+\cdots, P_{2}(z) \equiv z^{v}+\cdots$, each of the functions $P_{1}(z), P_{2}(z), Q_{1}(z), Q_{2}(z)$ is bounded and bounded from zero on $E$ for $n$ sufficiently large. We have

$$
\Phi^{\prime}(z) \equiv \frac{Q_{1} P_{1}^{\prime}-Q_{1}^{\prime} P_{1}}{Q_{1}^{2}}-\frac{Q_{2} P_{2}^{\prime}-Q_{2}^{\prime} P_{2}}{Q_{2}^{2}},
$$

so by the following additional inequalities on any circular disk of fixed radius in $E$ and hence for $z$ on $E$

$$
\begin{aligned}
\left|P_{1}(z)\right| \leqq p_{1}, & \left|P_{1}^{\prime}(z)\right| \leqq L p_{1} \nu, & \left|P_{2}(z)\right| \leqq p_{2}, & \left|P_{2}^{\prime}(z)\right| \leqq L p_{2} \nu, \\
\left|Q_{1}(z)\right| \leqq q_{1}, & \left|Q_{1}^{\prime}(z)\right| \leqq L q_{1} n, & \left|Q_{2}(z)\right| \leqq q_{2}, & \left|Q_{2}^{\prime}(z)\right| \leqq L q_{2} n ;
\end{aligned}
$$

as in Lemma 2 we deduce for $n$ sufficiently large

$$
\max \left|\Phi^{\prime}(z)\right| \leqq A_{1} n[\max |\Phi(z)|] \leqq A n .
$$

This is the analogue of (28).

The method of proof of Theorem 12 yields also

THEOREM 13. With the hypothesis of Theorem 12, the conclusion of Theorem 12 persists with the modifications that (47), (48), and (49) are replaced respectively by

$$
\begin{aligned}
\left\|F(z)-R_{v n}(z)\right\|_{q}^{\prime} & \leqq A / n^{k+\alpha} \tau^{n}, \quad q>0, \\
\left\|F(z)-R_{v n}(z)\right\|_{\infty} & \leqq A / n^{k+\alpha-2 / q} \tau^{n}, \\
\left|F(z)-R_{v n}(z)\right| & \leqq A \sigma^{n} / n^{k+\alpha-2 / q} \tau^{n}, \quad \text { z on } S,
\end{aligned}
$$

and if $k+\alpha-1-2 / q>0$ that (50) is replaced by

$$
\left|f(z)-1 / R_{v n}(z)\right| \leqq A / n^{k+\alpha-1-2 / q}, \quad z \text { on } \Gamma_{\tau} .
$$

It may be noted that in Theorems 12 and 13, as in Theorem 5, we have chosen $E$ to satisfy rather restrictive conditions. The reason is threefold. First, unless $E$ is bounded by analytic or other smooth curves, inequality (18) does not follow. Second, unless $E$ has an interior, and is bounded by relatively smooth curves, the 
method of Jackson does not apply. Third, it is not known at the outset whether the given $R_{v n}(z)$ have limit points of poles on $E$, but if so the reasoning used in connection with approximation on $E^{\prime}$ is valid only if $E$ has interior points; the $R_{v n}(z)$ have an unbounded number of possible finite poles.

The study of approximation by functions of type $(\nu, n)$ on point sets of class $A$ or class $B$, in the spirit of Theorems 11,12 , and 13 , presents no new difficulties, although further restrictions on the point sets may be necessary, and is left to the reader.

It is of some interest to note that the introduction of a weight function in the norms (1), (2), (3) throughout this paper presents no difficulty whatever. It is natural to assume that the weight function is bounded and bounded from zero, and integrable in the cases (2) and (3). Even boundedness and boundedness from zero can be lightened somewhat, as in $[5, \S 5.7]$.

If the function $f(z)$ is merely continuous on a closed bounded set $E$ which is dense in itself, a rational function $R_{n v}(z)$ of each type $(n, \nu)$ exists, of best approximation to $f(z)$ on $E$, as measured by (1), (2), or (3) as applicable. For each norm these rational functions (although not necessarily unique) can be arranged in a table

$$
\begin{aligned}
& R_{00}(z), R_{10}(z), R_{20}(z), \ldots, \\
& R_{01}(z), R_{11}(z), R_{21}(z), \ldots, \\
& R_{02}(z), R_{12}(z), R_{22}(z), \ldots,
\end{aligned}
$$

The present paper is devoted to the convergence of the rows of the table under certain conditions, and dually to the convergence of the columns of the table; convergence of diagonal and other sequences remains to be studied $\left({ }^{3}\right)$.

\section{REFERENCES}

1. J. L. Walsh, Note on the convergence of approximating rational functions of prescribed type, Proc. Nat. Acad. Sci. U.S.A. 50 (1963), 791-794.

2. - The convergence of sequences of rational functions of best approximation, Math. Ann. 155 (1964), 252-264.

3. - The convergence of sequences of rational functions of best approximation. II, Trans. Amer. Math. Soc. 116 (1965), 227-237.

$\left(^{3}\right)$ The present paper is devoted primarily to the study of Problem $\beta$, namely the study of the relationship between approximation on $E$ with geometric degree of convergence (and its refinements) on the one hand, and behavior of the function approximated interior to some $E_{\rho}$ (and on $E_{\rho}$ ) on the other hand. Problem $\alpha$ is the study of the relationship between degree of approximation on $E$ and properties on $E$ of the function approximated. Inequalities (18) and (19) are typical of Problem $\beta$; if $f(z)$ belongs to one of a large number of classes of functions on $E$, so also does [9] the product of $f(z)$ with an analytic function $\pi(z)$, so for suitably chosen polynomials $p_{n}(z)$ of respective degrees $n$ we deduce an inequality of form (18), and hence one of form (19). 
4. - The convergence of sequences of rational functions of best approximation with some free poles, Symposium on Approximation, 1964, General Motors Corp., Detroit, Mich., Ed. by H. L. Garabedian, Elsevier, Amsterdam, pp. 1-16.

5. —_, Interpolation and approximation, Colloq. Publ., Vol. 20, Amer. Math. Soc., Providence, R. I., 1935.

6. - An extension of the generalized Bernstein lemma, Colloq. Math. 16 (1967), 91-92.

7. - A sequence of rational functions with application to approximation by bounded analytic functions, Duke Math. J. 30 (1963), 177-190.

8. - Degree of polynomial approximation to an analytic function as measured by a surface integral. Proc. Nat. Acad. Sci. U.S.A. 48 (1962), 26-32.

9. J. L. Walsh and H. G. Russell, Integrated continuity conditions and degree of approximation by polynomials or by bounded analytic functions, Trans. Amer. Math. Soc. 92 (1959), 355-370.

10. D. Jackson, On certain problems of approximation in the complex domain, Bull. Amer. Math. Soc. 36 (1930), 851-857.

11. H. M. Elliott, On approximation to functions satisfying a generalized continuity condition, Trans. Amer. Math. Soc. 71 (1951), 1-23.

12. A. A. Gončar, On series of rational functions, Dokl. Akad. Nauk SSSR 143 (1962), 1246-1249 = Soviet Math. Dokl. 3 (1962), 562-565.

13. D. J. Newman, Rational approximation to $|x|$, Michigan Math. J. 11 (1964), 11-14.

UNIVERSITY OF MARYLAND, College Park, Maryland 